\title{
Exploratory biomarker assays: key assay parameters to evaluate in the face of evolving biomarker context-of-use
}

\author{
Sai P Thankamony*,1 \& Yan Zhang ${ }^{1}$ \\ ${ }^{1}$ Bioanalytical Sciences, Bristol-Myers Squibb Company, Princeton, NJ 08540, USA \\ *Author for correspondence: Sai.Thankamony@bms.com
“An exciting set of data can transform interest in an early exploratory biomarker into a key consideration for program-related decision-making"

First draft submitted: 10 September 2019; Accepted for publication: 14 October 2019; Published online: 28 November 2019

Keywords: biomarker $\bullet$ context of use $\bullet$ parallelism • repeatability $\bullet$ specificity

The field of biomarkers has seen extensive growth enabled by multiple advanced technological tools at our disposal. Such tools have enabled quantitative and semi-quantitative measurement of protein analytes in soluble matrices such as blood (serum or plasma), urine and cerebrospinal fluid. Soluble biomarkers are routinely analyzed using ligandbinding assay (LBA) and LC-MS/MS-based approaches, however LBA-based biomarker assays will be the focus of this discussion. The field of LBA has evolved from plate-based ELISA to now multiple options for ultrasensitive platforms such as Simoa ${ }^{\circledR}$ and SMCxPro ${ }^{\circledR}$. Despite assay validation guidelines for bioanalytical assays [1-3], inherent challenges of establishing biomarker assays make it an undertaking to follow a harmonized approach. One of the many challenges in biomarker assay development lies in the lack of true reference standard. Unlike pharmacokinetic (PK) assays, the calibrant material used for biomarker assays may not represent the endogenous analyte. Oftentimes, in biomarker assays, recombinant proteins with different physicochemical properties than the endogenous analyte are used instead. Additional challenges lie in inadequate and insufficient understanding of the biology of biomarker, such as presence and relevance of multiple isoforms, protein truncations, change in biomarker with disease state and lack of relevant samples during assay development.

\section{Biomarker assay validation}

Biomarker assays generally follow a fit-for-purpose validation (FFP) driven by the biomarker's context of use $(\mathrm{COU})[3,4]$. To state it simply, the level of assay validation is determined by the end use of the biomarker data. However, in the exciting and ever-evolving field of biomarker research, an initial set of data generated as part of a purely exploratory effort may inflate into a substantial interest beyond exploratory, thereby changing its COU. For example, data generated from a multiplex cytokine panel originally intended for biomarker discovery during early-stage clinical development may lead to identification of key biomarkers that become essential components of program-related decision making. Despite existence of assay development and validation guidance documents, due to the FFP validation practice for biomarkers, differences may exist across analysts either due to organizational practices or due to inherent challenges of developing a biomarker assay. Therefore, definition of what constitutes a COU-driven FFP validation may be left at the discretion of a technical expert. Often in biomarker assays, such practices may include lack of assay performance data collected using endogenous samples and sole reliance on standard curve performance and use of spiked quality control (QC) samples, both generated using purified recombinant or synthetic protein. In situations where an exploratory biomarker has progressed beyond exploratory and thereby has evolved in its COU, it may then become necessary to retrace the initial assay validation steps and re-establish parameters to define biomarker assay performance and qualification criteria. This process may not only be time consuming but, based on the initial level of validation performed (or lack thereof), may lead to unwanted surprises as well (e.g., discovery of assay cross-reactivity). A recommendation for exploratory biomarker 
assays that are on an FFP validation path is therefore to involve guided but versatile steps, even during the infantile interrogation of a biomarker.

\section{Charting the path on biomarker assay method development}

A close collaboration between the biomarker strategy-setting scientists and technology experts will allow the right questions to be asked at the onset of any assay development effort, thereby setting the right FFP validation criteria driven by the COU of the biomarker.

Regardless of intended application of biomarker data, one of the most important decisions in assay method development is critical reagent selection. Selection of critical reagents such as the calibrator material and reagent antibodies form a critical juncture for benchmarking assay quality. LBA biomarker assays are typically not absolute quantitation; however, they are relative quantitation to the calibrator material being utilized. Therefore, where possible effort must be made to select calibrator material that represents the endogenous form of the analyte [5]. Also, reagent antibodies must be selected for their ability to not just bind calibrator material but also endogenous material. Similarly, appropriate technological solutions should be utilized to characterize calibrator material as well as assay-associated reagent antibodies. Such technological solutions include MS to understand glycosylation patterns, amino acid sequence, length and purity [6]. Assay method development also involves determination of basic analytical performance such as LOD, LOQ, ULOQ and other factors such as QC selection and performance evaluation $[7,8]$. We hereby propose some additional but minimal method feasibility experiments for even highly exploratory biomarkers. Our recommendations come into effect after critical reagent selection but before initiating FFP assay validation. These recommendations can be followed when utilizing off-the-shelf commercial biomarker LBA kits as well.

\section{Minimal method feasibility recommendations}

For highly exploratory biomarkers undergoing a COU-driven FFP validation, it is critical to examine three additional parameters as part of method feasibility: parallelism, repeatability and specificity. Parallelism in biomarker assays allows understanding of dilution-response relationship of endogenous samples to the calibrator concentrationresponse curve [9]. Many publications have described how parallelism experiments should be run and what kind of data can be interpreted from these $[9,10]$. Recently, there has been tremendous focus on defining the difference between dilutional linearity and parallelism. Just as biomarker assays are not PK assays, dilutional linearity is not parallelism [4]. Where parallelism utilizes samples with endogenous analyte, dilutional linearity involves spiking sample matrix with known analyte and diluting into the standard curve range for recovery and linearity assessment [10]. Dilutional linearity may be an important element of biomarker assay development when the analyte of interest exists in very low endogenous levels (such as certain cytokines) or any other situation where it is not possible to obtain endogenous samples to undertake parallelism experiments. Parallelism experiments provide multifaceted assay performance information including specificity, minimal required dilution (MRD) and LLOQ [9-11]. Parallelism should be conducted using at least three to five individual samples and is one of the early set of key experiments to determine method feasibility. In situations where parallelism cannot be conducted due to lack of pertinent samples or parallelism fails during the course of evaluation, the assay may still be utilized based on the COU and utilized to generate data that is relative quantitation, provided assay can demonstrate repeatability.

Another key parameter for minimum method feasibility is therefore assay repeatability. Repeatability involves testing the same set of samples containing the endogenous analyte multiple times under similar testing conditions (such as same lab, operator and instrument) to obtain assay precision information. Repeatability experiments particularly become important when, as described above, for some reason parallelism cannot be demonstrated in an assay. Such assay repeatability experiments performed over a duration of time also provides information on assay-associated analytical variability. This analytical variability or assay noise is the extent to which an assigned nominal for an endogenous sample (such as an endogenous QC sample) can shift between multiple runs of same assay conditions. Data on assay-related variability or assay noise also enables a better understanding of what can be considered meaningful changes in biomarker levels. For example, in an assay, if the assigned nominal shifts $\pm 20 \%$ over multiple runs over a duration of period, it will give us confidence to interpret any changes beyond this $\pm 20 \%$ limit to be meaningful and attributable it to a biological event instead of an analytical observation.

The third key parameter to be determined during assay feasibility is assay specificity. Specificity is the ability to recognize the analyte of interest in the presence of other structurally similar molecules [3,7]. This parameter reiterates the need for a good understanding of the biomarker biology. Where possible, specificity experiments 
should also be performed early on and kept simple and relevant to the biomarker of interest. Specificity experiments are performed by spiking structurally close or variant forms of analyte of interest that may interfere with analyte detection (if physiologically relevant levels of such factors are known, then they should be tested at and above this level). Specificity experiments are key if close homologs of the analyte of interest are known to exist and, when generated early on, allows one to attribute the assay-related signal to the specific analyte of interest, thereby eliminating contributions or interference from such close homologs, resulting in generation of biologically relevant data.

\section{Conclusion}

An exciting set of data can transform interest in an early exploratory biomarker into a key consideration for program-related decision-making during clinical drug development. Therefore, following through these three essential parameters in parallelism, repeatability and specificity assessments are recommended for early exploratory biomarker assays. These three parameters should be evaluated along with routinely assessed analytical performance criteria. Testing these parameters for every exploratory assay may appear as resource constraining; however, there is superior advantage of garnering increased confidence in the assay early on. This would ensure that the right answers are provided to key questions raised during biomarker hypothesis generation. Incorporating these early assessments will also enable smooth transition of biomarker assays through their subsequent assay life cycle, such as clinical assay validation. Although FFP validation of biomarker assays are primarily driven by their COU, we must strive to gather parallelism, repeatability and specificity information during method feasibility, while allowing sufficient flexibility in this process so as to resourcefully support biological validation of the biomarker hypothesis.

\section{Financial \& competing interests disclosure}

The authors have no relevant affiliations or financial involvement with any organization or entity with a financial interest in or financial conflict with the subject matter or materials discussed in the manuscript. This includes employment, consultancies, honoraria, stock ownership or options, expert testimony, grants or patents received or pending, or royalties.

No writing assistance was utilized in the production of this manuscript.

\section{References}

1. Food and Drug Administration. Draft Guidance for Industry: Bioanalytical Method Validation. Food and Drug Administration, USA (2018). https://www.fda.gov/files/drugs/published/Bioanalytical-Method-Validation-Guidance-for-Industry.pdf

2. Piccoli SP, Sauer JM, Ackermann B et al. Points to consider document: scientific and regulatory considerations for the analytical validation of assays used in the qualification of biomarkers in biological matrices. Biomarker Assay Collaborative Evidentiary Considerations Writing Group, Critical Path Institute

(2019). https://c-path.org/wp-content/uploads/2019/06/evidconsid-whitepaper-analyticalsectionv2019.pdf

3. Lee JW, Devanarayan V, Barrett YC et al. Fit-for-purpose method development and validation for successful biomarker measurement. Pharm. Res. 23, 312-328 (2006).

4. Stevenson LF. Evolving our thinking on biomarker assay validation: are we ready for the next leap? Bioanalysis 11, 571-573 (2019).

5. Cowan KJ, Amaravadi L, Cameron MJ et al. Recommendations for selection and characterization of protein biomarker assay calibrator material. AAPS J. 19, 1550-1563 (2017).

6. Santockyte R, Zeng J, Zheng N. Critical reagent screening and characterization: benefits and approaches for protein biomarker assays by hybrid LC-MS. Bioanalysis 11, 785-795 (2019).

7. DeSilva B, Smith W, Weiner R et al. Recommendations for the bioanalytical method validation of ligand-binding assays to support pharmacokinetic assessments of macromolecules. Pharm. Res. 20, 1885-1900 (2003).

8. Valentin MA, Ma S, Zhao A et al. Validation of immunoassay for protein biomarkers: bioanalytical study plan implementation to support pre-clinical and clinical studies. J. Pharm. Biomed. Anal. 55(5), 869-877 (2011).

9. Stevenson LF, Purushothama S. Parallelism: considerations for the development, validation and implementation of PK and biomarker ligand-binding assays. Bioanalysis 6, 185-198 (2014).

10. Ciotti S, Purushothama S, Ray S. What is going on with my samples? A general approach to parallelism assessment and data interpretation for biomarker ligand-binding assays. Bioanalysis 5, 1941-1943 (2013).

11. Tu J, Bennett P. Parallelism experiments to evaluate matrix effects, selectivity and sensitivity in ligand-binding assay method development: pros and cons. Bioanalysis 9, 1107-1122 (2017). 
